\title{
Transport and deceleration of fusion products in microturbulence
}

\author{
George J. Wilkie, ${ }^{1,2}$ lan G. Abel, ${ }^{3}$ Matt Landreman, ${ }^{1}$ and William Dorland ${ }^{1}$ \\ ${ }^{1}$ University of Maryland, College Park, Maryland 20742, USA \\ ${ }^{2}$ Chalmers University, 41454 Gothenburg, Sweden \\ ${ }^{3}$ Princeton Center for Theoretical Science, Princeton, New Jersey 08544, USA
}

(Received 11 March 2016; accepted 22 May 2016; published online 6 June 2016)

The velocity-space distribution of alpha particles born in fusion devices is subject to modification at moderate energies due to turbulent transport. Therefore, one must calculate the evolution of an equilibrium distribution whose functional form is not known a priori. Using a novel technique, applicable to any trace impurity, we have made this calculation for fully nonlinear gyrokinetic simulations not only possible but also particularly efficient. We demonstrate a microturbulence-induced departure from the local slowing-down distribution, an inversion of the energy distribution, and associated modifications to the alpha heating and pressure profiles in an ITER-like scenario. Published by AIP Publishing. [http://dx.doi.org/10.1063/1.4953420]

Alpha particles are relied upon for heating a burning deuterium-tritium (DT) fusion reactor. ${ }^{1}$ The distribution of alpha particles in both radius and energy is therefore of critical importance for fusion. In addition to other important effects such as Alfvén eigenmodes, ${ }^{2-4}$ the ubiquitous microturbulence provides a background level of transport which has the potential to affect the radial and energy distribution of alpha particles. It is typically assumed that alpha particles slow down via collisions locally on a flux surface, but recent results ${ }^{5}$ suggest that this could be violated at moderate energies. In this case, the distribution function is not known a priori when performing turbulence simulations, and solving for the transport of a general non-Maxwellian distribution function would therefore be a computationally monumental task. Here, we assume alpha particles passively respond to the turbulence, an approximation that is widely used in this context. ${ }^{6-9}$ This socalled "trace" approximation is employed here to allow the fully turbulent transport to be solved extremely efficiently (about $1 \mathrm{~min}$ on a laptop, contrasted with tens of millions of CPU hours required without the trace approximation) when using specialized diagnostics from existing gyrokinetic simulations. Recently discovered effects ${ }^{10}$ close to the kinetic ballooning threshold notwithstanding, this approximation is generally a good one for alpha particles in microturbulence which is driven by primarily electrostatic modes. ${ }^{5,7}$

In the low-collisionality gyrokinetic hierarchy, the transport equation averaged over pitch angle reads ${ }^{11,12}$

$$
\frac{\partial F_{0 \alpha}}{\partial t}+\frac{1}{V^{\prime}} \frac{\partial}{\partial r} V^{\prime} \Gamma_{r}+\frac{1}{v^{2}} \frac{\partial}{\partial v} v^{2} \Gamma_{v}=S_{\alpha},
$$

where $V=V(r)$ is the volume enclosed by the flux surface labelled by $r$, the half-width at the height of the magnetic axis. $F_{0 \alpha}$ is the slowly varying distribution of alpha particles in the $r-v$ phase space. The energy-dependent source of alpha particles $S_{\alpha}$ is well-approximated by ${ }^{13,14} S_{\alpha} \propto \exp \left[-5 m_{\alpha}^{2}\left(v^{2}-v_{\alpha}^{2}\right) /\right.$ $\left.64 T_{i} E_{\alpha}\right]$ and has an overall magnitude so that the total source is that given in Ref. 15. The alpha particle mass is $m_{\alpha}$, the alpha birth energy is $E_{\alpha}=3.5 \mathrm{MeV}=m_{\alpha} v_{\alpha}^{2} / 2$, and $T_{i}$ is the temperature of the reactant ions. The radial flux due to turbulence is defined as

$$
\Gamma_{r} \equiv\left\langle\sum_{\sigma_{\|}} \int h_{\alpha} \mathbf{v}_{\chi} \cdot \nabla r \frac{\pi B \mathrm{~d} \lambda}{\sqrt{1-\lambda B}}\right\rangle_{t, \psi},
$$

where the pitch angle coordinate $\lambda \equiv E / \mu$ is the ratio between the energy and the magnetic moment, and $\sigma_{\|}$is the sign of the velocity parallel to the magnetic field (which has magnitude $B$ and points in the direction of the unit vector $\mathbf{b}$ ). The non-adiabatic part of the fluctuating alpha particle distribution is $h_{\alpha}$, and $\mathbf{v}_{\chi} \equiv \frac{C}{B} \mathbf{b} \times \nabla\langle\chi\rangle_{\mathbf{R}_{\chi}}$ characterizes the drift due to the turbulent electromagnetic potential $\chi \equiv \phi-\frac{v_{\|}}{c} A_{\|}$ (with $\phi$ and $A_{\|}$, respectively, the electrostatic and the parallel component of electromagnetic potentials). The notation $\langle\ldots\rangle_{t, \psi}$ signifies a time-average over many decorrelation times and a spatial average over a flux tube, and \langle\rangle$_{\mathbf{R}_{\alpha}}$ is the gyroaverage at fixed gyrocenter $\mathbf{R}_{\alpha}$. The flux in velocity space is defined as

$$
\begin{aligned}
\Gamma_{v} \equiv & -v \nu_{s} F_{0 \alpha}-\frac{1}{2} v^{2} \nu_{\|} \frac{\partial F_{0 \alpha}}{\partial v} \\
& +Z_{\alpha} e\left\langle\sum_{\sigma_{\|}} \int h_{\alpha}\left\langle\frac{\partial \chi}{\partial t}\right\rangle_{\mathbf{R}_{\alpha}} \frac{\pi B \mathrm{~d} \lambda}{\sqrt{1-\lambda B}}\right\rangle_{t, \psi}
\end{aligned}
$$

and includes both the test-particle energy scattering from the collision operator (with $\nu_{s}$ and $\nu_{\|}$are defined in Ref. 16, and summed over all bulk species) and the turbulent heating/ cooling of alpha particles. In this letter, we will make use of electrostatic gyrokinetic simulations in which $\chi=\phi$, but the framework is identical when electromagnetic fluctuations are accounted for.

The fluxes $\Gamma_{r}$ and $\Gamma_{v}$ are both expected to decrease rapidly with energy due to the large magnetic drift orbits and Larmor orbits of high-energy alpha particles. ${ }^{8,9,17}$ Therefore, high-energy alpha particles are expected to be well-confined with respect to microturbulence, while cooled-down helium in thermal equilibrium with the bulk plasma (i.e., "ash") transports similarly to the ions. At what energy this transition 
can be expected to occur and what the consequences are for alpha particle physics is the subject of this letter.

The evolution of the bulk plasma density and temperature takes place on the transport scale in the multiscale gyrokinetic hierarchy. ${ }^{18}$ Tools such as TRINITY ${ }^{19}$ and TGYRO ${ }^{20}$ have been developed to calculate the equilibrium profiles selfconsistently with microturbulent fluxes and have successfully recreated the experimental profiles of plasma density and temperature. However, these simulations are expensive (requiring about one million core hours for a single case) because of the need to repeatedly run turbulence simulations to steady-state. Global simulations that do not take advantage of the separation of scales are even more expensive.

In gyrokinetic simulations, the evolution of an additional species is usually as expensive as the bulk ions or electrons because the full distribution function still needs to be evolved regardless of relative density. This would make a transport calculation that much more expensive when impurities are included. Furthermore, if one does not know the form of the equilibrium distribution (as is the case for fast particles like alphas), an entire grid of $F_{0 \alpha}(v)$ at each flux surface is necessary to specify and adjust, as opposed to a small set of radially varying parameters (e.g., $n$ and $T$ for a Maxwellian). Therefore, if one were to include a trace nonMaxwellian species in TRINITY-like simulations, it would become at least a further order of magnitude more expensive. This letter outlines a method which improves upon this existing state-of-the-art by several orders of magnitude by taking advantage of the trace approximation.

One can schematically write the gyrokinetic equation for alpha particles in the form

$$
\mathcal{L}\left[h_{\alpha}\right]=-\frac{Z_{\alpha} e}{m_{s} v} \frac{\partial\langle\chi\rangle_{\mathbf{R}_{\alpha}}}{\partial t} \frac{\partial F_{\alpha 0}}{\partial v}-\mathbf{v}_{\chi} \cdot \nabla r \frac{\partial F_{0 \alpha}}{\partial r},
$$

where $\mathcal{L}$ is an operator that depends upon the fluctuating potential $\chi$ and represents the left-hand side of the gyrokinetic equation (which, in our case, does not include the socalled "parallel nonlinearity" ${ }^{21}$ ). Note that if alpha particles are trace in the sense that they do not affect the turbulence, then $h_{\alpha}$ contributes negligibly in the equations for $\chi$. In this case, the differential operator $\mathcal{L}$ is linear, in contrast to species which are not trace. If we invert Eq. (4) and insert $h_{\alpha}$ into Eq. (2), we find that the flux can be rigorously decomposed as

$$
\Gamma_{r}=-D_{r r} \frac{\partial F_{0 \alpha}}{\partial r}-D_{r v} \frac{\partial F_{0 \alpha}}{\partial v} .
$$

Similarly, the flux in velocity can be expressed as

$$
\Gamma_{v}=-v \nu_{s} F_{0 \alpha}-\left(\frac{1}{2} v^{2} \nu_{\|}+D_{v v}\right) \frac{\partial F_{0 \alpha}}{\partial v}-D_{v r} \frac{\partial F_{0 \alpha}}{\partial r},
$$

where the diffusion coefficients $D_{r r}, D_{r v}, D_{v r}$, and $D_{v v}$ have been introduced. A new code $\mathrm{T} 3 \mathrm{CORE}^{22}$ couples to existing nonlinear GS2 ${ }^{23,24}$ simulation output and uses a finite volume method to solve Eq. (1). With specialized diagnostics, GS2 is used to solve for the diffusion coefficients in Eqs. (5) and (6) by including two trace species with the same mass and charge as alpha particles, but with different $\partial F_{0} / \partial r$ and/ or $\partial F_{0} / \partial v$. Note these "test" species can even be Maxwellian, and that is enough to determine the diffusion coefficients (see a proof of principle in Ref. 5). It is remarkable that, with the use of these diffusion coefficients, one can rigorously recreate the turbulent flux of a trace species as if one ran another nonlinear gyrokinetic simulation.

In this letter, we focus on the transport of alpha particles in gyrokinetic microturbulence. However, the novel technique described in this letter is generally applicable to the global turbulent transport of any trace impurity. Further details about the T3CORE code will be provided in a forthcoming publication, but the source code is freely available online $^{22}$ for examination.

As a representative example of a D-T scenario of ITER, we used case 10010100 from the CCFE public database: ${ }^{25}$ a TRANSP simulation of an ELMy H-mode ${ }^{26}$ at 15 MA plasma current and a flat electron density profile. Using these data for the bulk plasma equilibrium, the flux tube code GS2 was used to calculate the local turbulence properties (including the alpha particle diffusion coefficients) driven by instability of the ion temperature gradient (ITG) mode at four radii in the range $0.5 \leq r / a \leq 0.8$ (see note ${ }^{27}$ ), where $a$ is the halfwidth of the separatrix. The intensity of the turbulence is characterized by the approximate ion diffusivity given by $q_{i}=-\chi_{i} n_{i} T_{i}^{\prime}(r)$, where $n_{i}, T_{i}$, and $q_{i}$ are the deuterium density, temperature, and heat flux respectively. This is shown along with the basic plasma properties in Table I from our simulations and is generally consistent with the previous computational $^{6,28}$ and experimental ${ }^{29,30}$ results. The active species are deuterium, tritium, and electrons in these electrostatic simulations.

Alpha particles produced in the region $0<r / a<0.5$ are assumed to enter the domain as Maxwellian ash at the local ion temperature. The distribution $F_{0}(r=0.8 a, v)$ at the outer edge of the domain is fixed to be the local slowing down distribution, ${ }^{31}$ plus a population of helium ash at the local ion temperature to bring the total helium density to $n_{\mathrm{He}}=10^{17} / \mathrm{m}^{3}$, approximately in agreement with the edge density in Ref. 26.

Using T3CORE, we can determine $F_{0 \alpha}$, the steady-state equilibrium distribution of alpha particles in the profile described above. The resulting distribution is shown in Fig. 1. A significant feature is the departure from the classical analytic slowing-down distribution ${ }^{31}$ (comparison shown in Fig. 2), including an inversion around $v / v_{\alpha} \sim 0.25$. Similar

TABLE I. Summary of basic profiles considered in this work. First three columns are from the CCFE public database, ${ }^{25}$ and the last column is calculated from GS2 simulation. The electron density is $10^{20} / \mathrm{m}^{3}$ for all radii, and the ion mix is half deuterium, half tritium, with $T_{D}=T_{T}=T_{i}$. The gyroBohm diffusivity is defined as $\rho_{c}^{2} c_{s} / a$, where $c_{s} \equiv \sqrt{T_{e} / m_{D}}$ is the sound speed of deuterium and is the speed with respect to which $\rho_{c}$ is defined.

\begin{tabular}{ccccc}
\hline \hline$r / a$ & $T_{i}(\mathrm{keV})$ & $T_{e}(\mathrm{keV})$ & $\rho_{D} / a$ & $\chi_{i} / \chi_{\mathrm{GB}}$ \\
\hline 0.5 & 13.4 & 16.0 & 0.0021 & 0.74 \\
0.6 & 10.9 & 12.9 & 0.0018 & 2.3 \\
0.7 & 8.4 & 9.7 & 0.0016 & 5.4 \\
0.8 & 5.8 & 6.6 & 0.0013 & 8.7 \\
\hline \hline
\end{tabular}




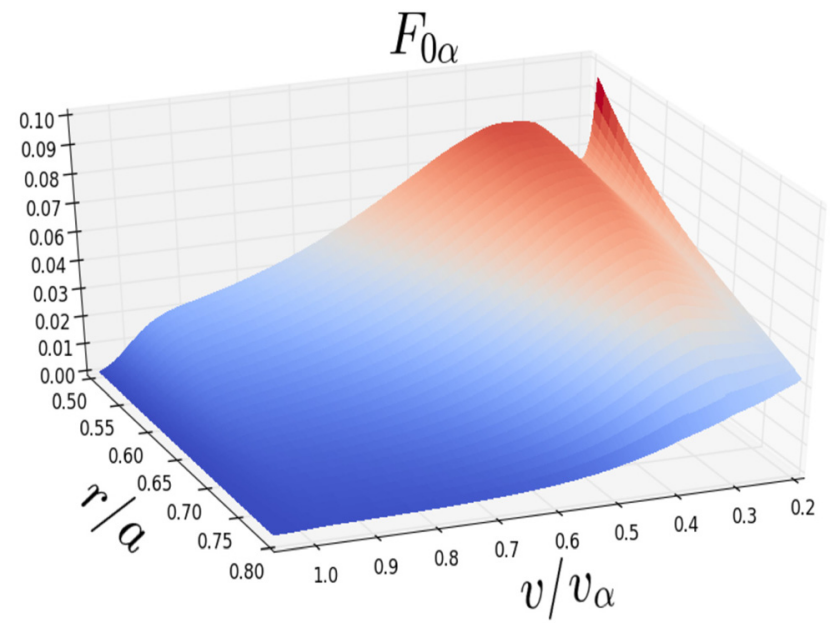

FIG. 1. The calculated alpha particle distribution from $\mathrm{T} 3 \mathrm{CORE}$ in the presence of fusion source, turbulence, and collisions, focusing on the highenergy tail (note there exists an approximately ion-temperature Maxwellian in the region $v<0.2 v_{\alpha}$.

inversions have been seen in JET D-T experiments, ${ }^{32}$ and previous analytic transport models. ${ }^{33}$ This inversion exists because the turbulent flux is a strong function of energy ${ }^{8}$ due to finite-orbit width effects, and the transport can be strong compared to collisions at moderate suprathermal energies, ${ }^{5}$ "carving out" that part of the distribution. At high energy, the collisional slowing-down time approaches an upper bound, while the transport time continues increasing, which makes the local slowing-down distribution a better approximation there.

The modified $F_{0 \alpha}$ found from simulation has an impact on several properties associated with alpha particles. First, the collisional plasma heating is shown in Fig. 3(a) to be adversely affected by the presence of turbulence due to the change in the alpha particle energy distribution. Furthermore, even though alpha particles have relatively low density, their pressure gradient can account for a significant fraction of that of the total plasma. Therefore, a change in the pressure profile such as shown in Fig. 3(b) can have a feedback effect on the magnetic geometry, and/or Alfvén eigenmodes.

If alpha particles escape the plasma at high energy, they have the potential to damage the plasma-facing components of a reactor. Therefore, an important question of alpha particle transport is if they slow down to sufficiently low energy before escaping the plasma. While we do not model the

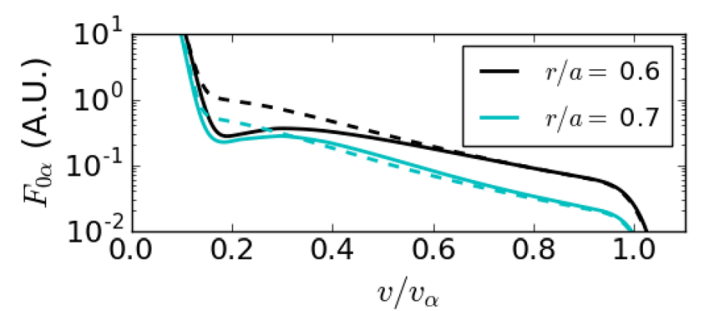

FIG. 2. Comparison at two internal radial grid points: $r=0.6 a$ (black) and $r=0.7 a$ (cyan) between the local alpha particle distribution as calculated with T3CORE (solid lines) and the analytic slowing down distribution (dashed lines). A population of Maxwellian ash was artificially added to the latter so that the total helium density is the same between the two. The ash makes up about $50 \%-70 \%$ of the total alpha particle density for these cases.

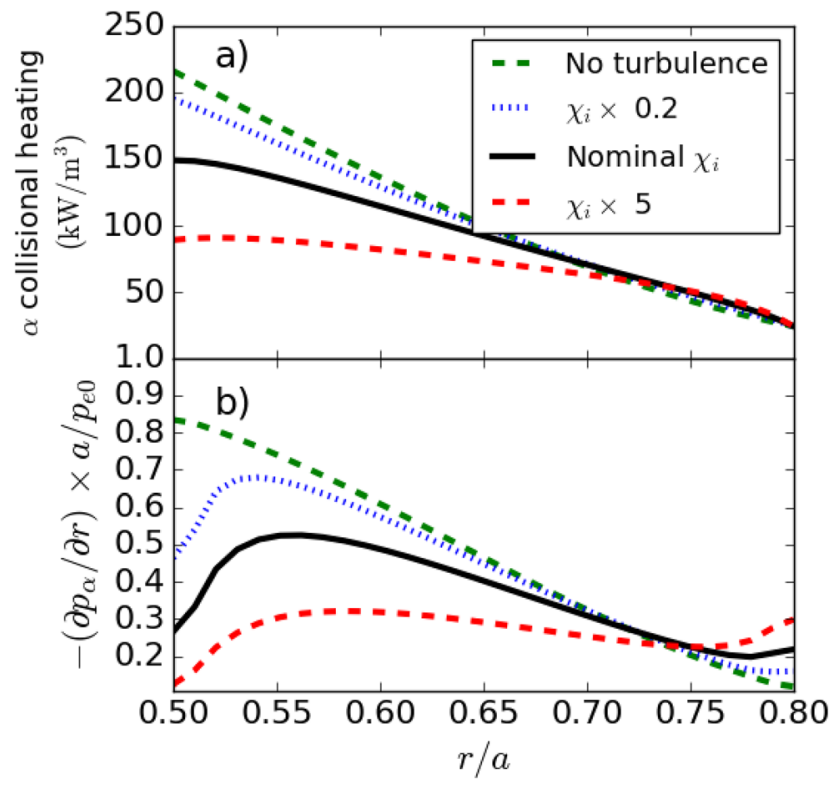

FIG. 3. Comparison of the radial profiles of: (a) bulk plasma collisional heating by alpha particles, and (b) alpha particle pressure gradient normalized to the electron pressure at the magnetic axis. Displayed are the results from the analytic local slowing-down distribution (green dashed) and cases from T3CORE where the nominal turbulent fluctuation amplitude and diffusion coefficients (black solid) are scaled up (red dashed) and down (blue dotted) by factors of five.

separatrix region, we can calculate the spectrum of alpha particles leaving the domain at $r=0.8 a$, and this is shown in Fig. 4.

The intensity of microturbulence is typically quite sensitive to the gradients of density and temperature (i.e., "stiff"), hence it is appropriate to examine the sensitivity of our results to the turbulence intensity in Figs. 3 and 4. There, we show the alpha particle heating, pressure, and heat flux profiles while scaling the turbulent diffusion coefficients higher and lower by a factor of five from the nominal case.

The results presented here show that at energies around $300 \mathrm{keV}$ for our ITER-like scenario, the alpha particle distribution is modified by the presence of ITG microturbulence, including an inversion that has been observed in other experiments. ${ }^{32}$ How strong this departure is from the classical slowing-down distribution depends on the details of the turbulence. Since the slowing-down distribution is mostly unaffected at high energy, we find that alpha particles largely do their job of heating the plasma, with some order unity corrections to the heating rate depending on the turbulence

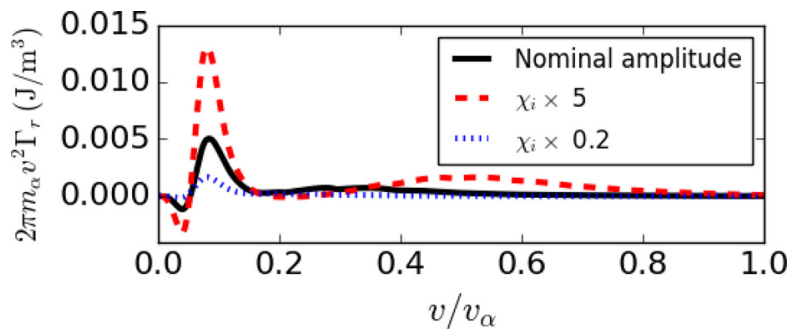

FIG. 4. Integrand of the alpha particle heat flux (which can be expressed as $\left.q_{\alpha}=\int\left(m_{\alpha} v^{2} / 2\right) \Gamma_{r} 4 \pi v^{2} \mathrm{~d} v\right)$ as calculated by T3CORE at $r=0.8 a$, showing the spectrum of alpha particles exiting the domain. 
amplitude. Also, the alpha particle pressure profile can be significantly modified, which can in turn affect the magnetic geometry. Furthermore, the stability of Alfvén eigenmodes has recently been shown to be very sensitive to the alpha particle pressure profile, ${ }^{34,35}$ so the flattening of the alpha particle pressure is beneficial in this context. Our results also indicate that turbulence has only a moderate effect on the alpha particle heat flux in the high-energy part of the distribution. Only when the amplitude of the turbulence is scaled up by a factor of five does the energetic alpha particle flux become significant around $1 \mathrm{MeV}$.

Our results come with some caveats. First, the trace approximation plays a central role in our analysis, and this could exclude some important effects. Also, the bulk equilibrium profiles were modelled from existing TRANSP simulations, which uses simple estimates for anomalous transport. An improved prediction would use equilibria calculated from TRINITY, TGYRO, or experimental profiles. This would allow for more robust studies of ash transport. For example, in this letter, we used a relatively low ash density to minimize the effect of an unphysical departure from the ion temperature (since the heat transport of the ash is calculated consistently with the turbulence while the ion profile is held fixed). The Gs2 simulations used here included only electrostatic fluctuations, and it is possible that Alfvénic fluctuations can significantly affect the alpha particle transport ${ }^{8}$ or underlying turbulence. ${ }^{10}$ Finally, even though alpha particles are born isotropically in velocity space, there is the possibility that turbulence induces anisotropy in the velocity distribution, a possibility our analysis excludes.

This letter demonstrated the importance of microturbulence to the distribution of alpha particles in an ITER-like scenario and the associated consequences. The computational tool developed to solve this problem, T3CORE, can be coupled as a module to any of the other existing gyrokinetic or transport tools without even the need to generalize the former for non-Maxwellian distributions. With the ability to efficiently and rigorously model alpha particles in turbulence, the fusion community can make more accurate and routine predictions for the performance of ITER and devices beyond.

The authors would like to thank Alex Schekochihin, Michael Barnes, Greg Hammett, and Felix Parra for helpful discussions. Workshops hosted by the Wolfgang Pauli Institute in Vienna and CIEMAT in Madrid were critical to the success of this project.

This work was supported by the U.S. Department of Energy by Grant Nos. DE-FG02-93ER54197 and DE-FC0208ER54964. The nonlinear GS2 simulations were performed on the supercomputer Hopper at NERSC.

\footnotetext{
${ }^{1}$ A. Fasoli, C. Gormenzano, H. L. Berk, B. Breizman, S. Briguglio, D. S. Darrow, N. Gorelenkov, W. W. Heidbrink, A. Jaun, S. V. Konovalov, R. Nazikian, J.-M. Noterdaeme, S. Sharapov, K. Shinohara, D. Testa, K. Tobita, Y. Todo, G. Vlad, and F. Zonca, "Chapter 5: Physics of energetic ions," Nucl. Fusion 47(6), S264 (2007).

${ }^{2}$ G. Y. Fu and J. W. Van Dam, "Excitation of the toroidicity-induced shear Alfvén eigenmode by fusion alpha particles in an ignited tokamak," Phys. Fluids B 1(10), 1949 (1989).
}

${ }^{3}$ B. N. Breizman and S. E. Sharapov, "Energetic particle drive for toroidicity-induced Alfven eigenmodes and kinetic toroidicity-induced Alfven eigenmodes in a low-shear tokamak," Plasma Phys. Controlled Fusion 37, 1057 (1995).

${ }^{4}$ S. Günter, G. Conway, S. daGraça, H.-U. Fahrbach, C. Forest, M. Garcia Muñoz, T. Hauff, J. Hobirk, V. Igochine, F. Jenko, K. Lackner, P. Lauber, P. McCarthy, M. Maraschek, P. Martin, E. Poli, K. Sassenberg, E. Strumberger, G. Tardini, E. Wolfrum, H. Zohm, and ASDEX Upgrade Team, "Interaction of energetic particles with large and small scale instabilities," Nucl. Fusion 47(8), 920 (2007).

${ }^{5}$ G. Wilkie, I. Abel, E. Highcock, and W. Dorland, "Validating modeling assumptions of alpha particles in electrostatic turbulence," J. Plasma Phys. 81, 905810306 (2015)

${ }^{6}$ C. Estrada-Mila, J. Candy, and R. E. Waltz, "Turbulent transport of alpha particles in reactor plasmas," Phys. Plasmas 13, 112303 (2006).

${ }^{7}$ C. Angioni and A. G. Peeters, "Gyrokinetic calculations of diffusive and convective transport of $\alpha$ particles with a slowing-down distribution function," Phys. Plasmas 15, 052307 (2008).

${ }^{8}$ T. Hauff, M. J. Pueschel, T. Dannert, and F. Jenko, "Electrostatic and magnetic transport of energetic ions in turbulent plasmas," Phys. Rev. Lett. 102, 075004 (2009).

${ }^{9}$ M. J. Pueschel, F. Jenko, M. Schneller, T. Hauff, S. Günter, and G. Tardini, "Anomalous diffusion of energetic particles: Connecting experiment and simulations," Nucl. Fusion 52, 103018 (2012).

${ }^{10}$ J. Citrin, F. Jenko, P. Mantica, D. Told, C. Bourdelle, J. Garcia, J. W. Haverkort, G. M. D. Hogeweij, T. Johnson, and M. J. Pueschel, "Nonlinear stabilization of tokamak microturbulence by fast ions," Phys. Rev. Lett. 111, 155001 (2013).

${ }^{11}$ R. E. Waltz, E. M. Bass, and G. M. Staebler, "Quasilinear model for energetic particle diffusion in radial and velocity space," Phys. Plasmas 20 042510 (2013).

${ }^{12}$ G. Wilkie, "Microturbulent transport of non-Maxwellian alpha particles," Ph.D. thesis, University of Maryland, 2015.

${ }^{13}$ H. Brysk, "Fusion neutron energies and spectra," Plasma Phys. 15(7), 611 (1973).

${ }^{14} \mathrm{~B}$. Appelbe and J. Chittenden, "The production spectrum in fusion plasmas," Plasma Phys. Controlled Fusion 53(4), 045002 (2011).

${ }^{15}$ J. Huba, NRL Plasma Formulary (Naval Research Laboratory, Washington, 2011).

${ }^{16} \mathrm{P}$. Helander and D. Sigmar, Collisional Transport in Magnetized Plasmas (Cambridge University Press, 2002).

${ }^{17}$ W. Zhang, V. Decyk, I. Holod, Y. Xiao, Z. Lin, and L. Chen, "Scalings of energetic particle transport by ion temperature gradient microturbulence," Phys. Plasmas 17, 055902 (2010).

${ }^{18}$ I. Abel, G. G. Plunk, E. Wang, M. Barnes, S. C. Cowley, W. Dorland, and A. Schekochihin, "Multiscale gyrokinetics for rotating tokamak plasmas: Fluctuations, transport and energy flows," Rep. Prog. Phys. 76, 116201 (2013).

${ }^{19}$ M. Barnes, I. Abel, W. Dorland, T. Görler, G. W. Hammett, and F. Jenko, "Direct multiscale coupling of a transport code to gyrokinetic turbulence codes," Phys. Plasmas 17, 056109 (2010).

${ }^{20}$ J. Candy, C. Holland, R. E. Waltz, M. R. Fahey, and E. Belli, "Tokamak profile prediction using direct gyrokinetic and neoclassical simulation," Phys. Plasmas 16, 060704 (2009).

${ }^{21}$ J. Candy, R. E. Waltz, S. E. Parker, and Y. Chen, "Relevance of the parallel nonlinearity in gyrokinetic simulations of tokamak plasmas," Phys. Plasmas 13, 074501 (2006)

${ }^{22}$ See http://www.github.com/gjwilkie/t3core for the Julia source code.

${ }^{23}$ M. Kotschenreuther, G. Rewoldt, and W. M. Tang, "Comparison of initial value and eigenvalue codes for kinetic toroidal plasma instabilities," Comput. Phys. Commun. 88, 128 (1995).

${ }^{24}$ W. Dorland, F. Jenko, M. Kotschenreuther, and B. N. Rogers, "Electron temperature gradient turbulence," Phys. Rev. Lett. 85, 5579 (2000).

${ }^{25}$ C. Roach, M. Walters, R. Budny, F. Imbeaux, T. Fredian, M. Greenwald, J. Stillerman, D. Alexander, J. Carlsson, J. Cary, F. Ryter, J. Stober, P. Gohil, C. Greenfield, M. Murakami, G. Bracco, B. Esposito, M Romanelli, V. Parail, P. Stubberfield, I. Voitsekhovitch, C. Brickley, A. R. Field, Y. Sakamoto, T. Fujita, T. Fukuda, N. Hayashi, G. M. D. Hogeweij, A. Chudnovskiy, N. A. Kinerva, C. E. Kessel, T. Aniel, G. T. Hoang, J. Ongena, E. J. Doyle, W. A. Houlberg, A. R. Polvoi, ITPA Confinement Database, Modelling Topical Group, and ITPA Transport Physics Topical Group, "The 2008 public release of the international multi-tokamak confinement profile database," Nucl. Fusion 48, 125001 (2008). 
${ }^{26}$ R. Budny, "Fusion alpha parameters in tokamaks with high DT fusion rates," Nucl. Fusion 42, 1383 (2002).

${ }^{27}$ This annulus was chosen because ITG was found to be stable below $r<$ $0.5 a$ for this profile, and we wish to avoid the edge where ripple loss or direct orbit loss of alphas is appreciable. Yet, a significant fraction of the total alpha particles are produced in this region.

${ }^{28}$ A. M. Dimits, G. Bateman, M. Beer, B. I. Cohen, W. Dorland, G. W. Hammett, C. Kim, J. E. Kinsey, M. Kotschenreuther, A. H. Kritz, L. L. Lao, J. Mandrekas, W. M. Nevins, S. E. Parker, A. J. Redd, D. E. Shumaker, R. Sydora, and J. Weiland, "Comparisons and physics basis of tokamak transport models and turbulence simulations," Phys. Plasmas 7, 969 (2000).

${ }^{29}$ R. J. Groebner, W. Pfeiffer, F. P. Blau, K. H. Burrell, E. S. Fairbanks, R. P. Seraydarian, H. St. John, and R. E. Stockdale, "Experimentally inferred ion thermal diffusivity profiles in the Doublet III Tokamak: Comparison with neoclassical theory," Nucl. Fusion 26, 543 (1986).

${ }^{30}$ S. D. Scott, P. H. Diamond, R. J. Fonck, R. J. Goldston, R. B. Howell, K. P. Jaehnig, G. Schilling, E. J. Synakowski, M. C. Zarnstorff, C. E. Bush,
E. Fredrickson, K. W. Hill, A. C. Janos, D. K. Mansfield, D. K. Owens, H. Park, G. Pautasso, A. T. Ramsey, J. Schivell, G. D. Tait, W. M. Tang, and G. Taylor, "Local measurements of correlated momentum and heat transport in the TFTR tokamak," Phys. Rev. Lett. 64(5), 531 (1990).

${ }^{31}$ J. D. Gaffey, "Energetic ion distribution resulting from neutral beam injection in tokamaks," J. Plasma Phys. 16, 149 (1976).

${ }^{32}$ JET Team, "Alpha particle studies during JET DT experiments," Nucl. Fusion 39, 1619 (1999).

${ }^{33}$ D. J. Sigmar, R. Gormley, and G. Kamelander, "Effects of anomalous alpha particle diffusion on fusion power coupling into tokamak plasma," Nucl. Fusion 33(5), 677 (1993).

${ }^{34} \mathrm{Ph}$. Lauber, "Local and global kinetic stability analysis of Alfvén eigenmodes in the 15 MA ITER scenario," Plasma Phys. Controlled Fusion 57(5), 054011 (2015).

${ }^{35}$ P. Rodrigues, A. Figueiredo, J. Ferreira, R. Coelho, F. Nabais, D. Borba, N. F. Loureiro, H. J. C. Oliver, and S. E. Sharapov, "Systematic linearstability assessment of Alfvén eigenmodes in the presence of fusion $\alpha$-particles for ITER-like equilibria," Nucl. Fusion 55(8), 083003 (2015). 